\section{Paleopatología osteoarticular en Chinchorro. Revisión de un caso y discusión sobre el autocuidado en la prehistoria de Arica, norte de Chile}

\author{
VERÓNICA SILVA-PINTO ${ }^{1, a, b}$, DANIELA VALENZUELA ${ }^{2, c}$, \\ MARCELA SEPÚLVEDA ${ }^{1, d}$
}

\section{Osteoarticular paleopathology in a Chinchorro body. Report of one case}

We report a severe osteoarthropathy in a body of a Chinchorro adult male exhumed from the pre-Hispanic site of Playa Miller 8, located in the city of Arica, on the northern coast of Chile. The advanced state of joint disease shows that the individual was cared by his social group, to be able to survive. This care was perpetuated in the postmortem treatment, applying red color to the body. Particular self-care social dynamics of Chinchorro society are inferred.

(Rev Med Chile 2013; 141: 637-643).

Key words: Empathy; Chinchorro; Mummies; Osteoarthritis.

'Departamento de Antropología,
Universidad de Tarapacá.
${ }^{2}$ Instituto de Investigaciones
Arqueológicas, Universidad Católica
del Norte.
aAntropóloga Física, Doctora (c) en
Antropología.
'Becaria CONICYT. Programa de
postgrado UTA-UCN.
'Arqueóloga, Doctora (c) en
Antropología.
dArqueóloga, Doctora en Prehistoria.
Trabajo financiado por: Proyecto
FONDECYT
No 1100354 Tecnología, función
y significado del uso de colores
en poblaciones arcaicas del
extremo norte de Chile. Beca
Doctorado Nacional 2010 CONICYT
N²1100628.
Recibido el 29 de abril de 2012,
aceptado el 8 de febrero de 2013.
Corrrespondencia a:
Verónica Silva-Pinto
Casilla 6-D, Arica, Chile.
Teléfono: $56-58-2319628$
Fax: $56-58-2224248$
E-mail: vsilvap@uta.cl

1 xiste la creencia generalizada que entre grupos cazadores recolectores, las personas que requerían de cuidados especiales, tales como discapacitados o ancianos, eran eliminados o abandonados ${ }^{1}$. Sin embargo, el autocuidado entendido como toda actividad realizada por personas, familias o grupos sociales para fomentar, mantener y limitar daños a la salud, que incluye el cuidado de los enfermos, corresponde a una práctica que ha acompañado a los seres humanos desde su origen y ha permitido la sobrevivencia de la especie ${ }^{2,3}$.

En este trabajo presentamos un caso de osteoartropatía poliarticular severa en un cuerpo adulto, rescatado en el sitio Playa Miller 8 (PLM-8, norte de Chile), perteneciente a la tradición Chinchorro del período Arcaico (10.500-3.500 A.P. $)^{4}$ (Figura 1). Debido a que al momento de morir el individuo presentaba un severo cuadro de deformidad y rigidez articular, se propone que debió recibir cuidados especiales por parte de su grupo para subsistir. Su integración como miembro del grupo social se refleja también en el tratamiento postmortem del cuerpo.

Las osteoartropatías más comunes correspon- den a las degenerativas que afectan principalmente a las articulaciones sinoviales debido a la pérdida gradual del cartílago articular y la subsecuente exposición de la superficie del hueso subcondral, provocando contacto interóseo y deformación articular $^{4-6}$. Estas lesiones se manifiestan con dolor crónico en las articulaciones y una progresiva incapacidad funcional, la que puede producirse en un lapso de pocos meses a 20 años de evolución, con una consecuente invalidez e incluso mortalidad prematura ${ }^{4}$.

Las osteoartropatías degenerativas denominadas también osteoartrosis u osteoartritis en la bibliografía inglesa, se diferencian en primaria y secundaria. La más frecuente es la primaria o idiopática, que no va precedida de otra patología, siendo la edad y el estrés mecánico de las articulaciones una de las causas más recurrentes. Las artropatías secundarias en cambio son consecuencia de una enfermedad previa, ya sea de carácter congénito o adquirido, es decir, como consecuencia de displasia o deformidades congénitas, traumas, enfermedades metabólicas, sistémicas, nutricionales, vasculares, neurotróficas, tóxicas e infecciosas 
entre otras, o secundaria a otro tipo de artropatía como la artritis reumatoide o la artritis juvenil ${ }^{5,7-10}$.

La osteoartrosis es conocida como la enfermedad más antigua del mundo, ya que se demuestra su presencia en animales desde la Era Mesozoica $(251,0 \pm 0,4-65,5 \pm 0,3 \mathrm{Ka})$ con las mismas características histológicas que lo observado en la actualidad. Su ocurrencia en el género Homo se evidencia a partir del Homo erectus hace 1,8 millones de años ${ }^{4}$.

En paleopatología, es posible detectar osteartropatías a través de observación directa de las superficies articulares de los huesos, con lesiones que se caracterizan por eburnación, quistes y/o esclerosis subcondral, porosidad y aposición ósea secundaria marginal o central (osteofitos) ${ }^{4,7-10}$. No obstante, su diagnóstico y etiología son difíciles de determinar, agrupando a enfermedades tan diversas como la artrosis deformante, artritis reumatoide, artritis juvenil y varias osteartropatías secundarias, lo que contribuye a la alta frecuencia de reportes de casos $^{6-9}$.

\section{Caso de estudio}

Por motivo de la construcción del Edificio Punta del Este, frente a la playa El Laucho en el sector residencial sur de la ciudad de Arica, se realizó el hallazgo de un entierro primario disturbado, del que se rescató un cuerpo humano esqueletizado incompleto de la tradición Chinchorro.
El cuerpo, clasificado como PLM-8PEC1, se encontraba bajo una estera de fibra vegetal tejida, en posición decúbito dorsal extendido, orientación sacro-vértex sureste-noroeste y con los antebrazos en pronación (palmas hacia abajo). El esqueleto se halló en regular estado de conservación, con algunos huesos estables y otros frágiles y quebradizos. Afloramientos de sales en la superficie ósea serían la principal causa del agrietamiento del tejido óseo cortical y su fragmentación. Como parte del tratamiento postmortem, el cuerpo presenta pigmento rojo sobre la piel de la cabeza, pecho y espalda.

La caracterización elemental físico-química, mediante SEM-EDX, de una muestra de pigmento de la cabeza, identifica el uso de óxidos de hierro con formas de agujas (posiblemente hematita) mezclados a otros componentes inorgánicos minoritarios (sílice, calcio y sodio) (Figura 2). El fechado radiocarbónico por C14 AMS de la estera presente sobre el cuerpo, arrojó una edad calibrada de $4.170 \pm 40$ A.P. (Beta 286650; fibra vegetal), coincidente con otra obtenida previamente en PLM-8 de $4.572 \pm 105$ cal. A.P ${ }^{11}$.

El análisis bioantropológico basado en métodos y técnicas estándares ${ }^{12}$ permitió determinar que PLM-8PEC1 corresponde a un individuo de sexo masculino, con una edad al morir de 30 a 35 años y una estatura de 1,63 $\pm 0,4 \mathrm{~m}$. El análisis paleopatológico se realizó a través de la observación morfoscópica del tejido óseo, evidenciando exostosis auditiva externa bilateral, desgaste severo de la dentadura del maxilar con destrucción completa

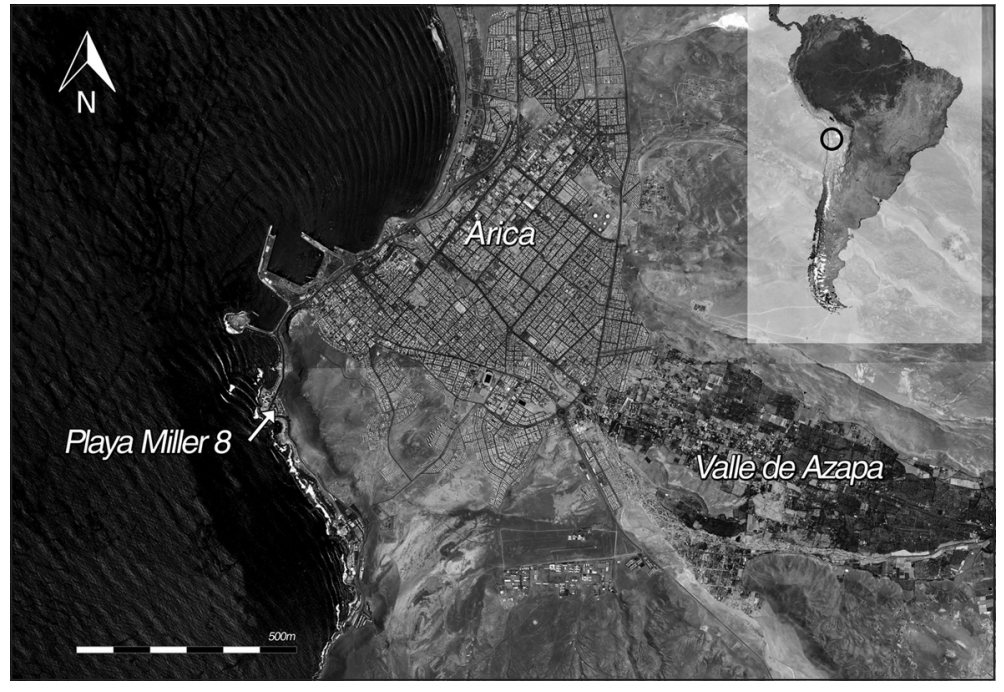

Figura 1. 

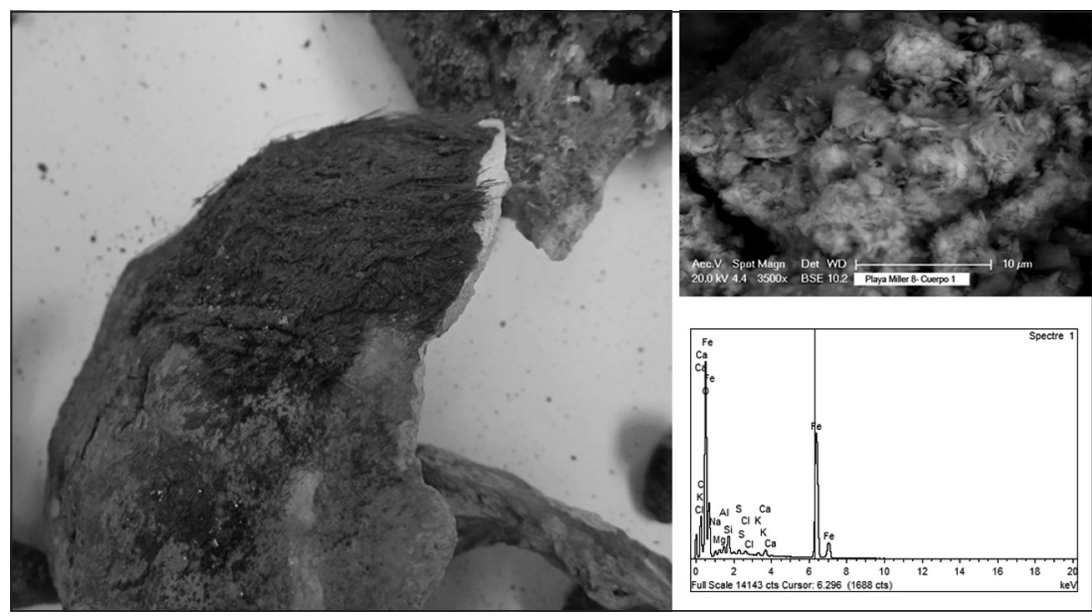

Figura 2.

de la corona y pérdida en vida de todos los dientes de la mandíbula con excepción de las piezas 38,47 y 48 (FDI). A esto se suma la presencia de artropatía poliarticular severa que afecta principalmente a la columna vertebral, codos, muñecas y manos de forma bilateral y la rodilla derecha (Figura 3).

- En las vértebras cervicales C2 y C3 se presenta eburnación con marcada porosidad y ampliación de las carillas articulares. En las vértebras lumbares se observa degeneración articular y osteofitosis de grado severo en L1-L2 y moderado en L3 a L5. Esta última presenta además espondilolisis completa (bilateral).

- En la articulación humeroradioulnar (codo) derecha existe eburnación, degeneración articular y osteofitosis severa en la tróclea humeral, la fosa olecraneana y coronoides, que limitó el movimiento de extensión y flexión del antebrazo. Se presentan además foraminas periarticulares (vestigio de probables abscesos) en la región lateral y medial del olécranon de la ulna, evidenciando procesos infecciosos y necrosis del tejido óseo.

- La articulación radioulnar distal y radiocarpiana de ambas extremidades exhiben eburnación y degeneración articular. La epífisis distal de la ulna izquierda evidencia foraminas periarticulares. Tanto la epífisis distal del radio como el hueso escafoides izquierdo muestran osteofitos moderados y deformación articular.

Pese a conservarse sólo fragmentos de los fémures, la epífisis distal del fémur derecho manifiesta eburnación severa con surcos por el desgaste cortical del cóndilo lateral y osteofitos moderados hacia medial y lateral.

\section{Discusión}

La prehistoria del norte de Chile se remonta a más de 10.000 años durante la transición Pleistoceno-Holoceno, correspondiente en arqueología al denominado período Paleoindio ${ }^{13}$. En el consecutivo período Arcaico (10.500-3.500 A.P.), la zona costera del extremo norte del país es poblada por grupos Chinchorros caracterizados como cazadores, pescadores y recolectores con una adaptación esencialmente marítima y desarrolladas técnicas de pesca ${ }^{14-18}$. Destaca en estos grupos, a partir de 8.000 años ca. A.P., la momificación artificial de infantes y adultos mediante complejos tratamientos de los cuerpos, con su evisceración, relleno y recubrimiento ${ }^{15}$. La variedad de tratamientos identificados incluye desde el modelado del cuerpo a modo de escultura, hasta cuerpos sin tratamientos artificiales y recubiertos parcialmente de pigmento rojo. No obstante esta variabilidad, todos los entierros se presentan en posición decúbito dorsal extendido, cubiertos o envueltos en mortajas de estera vegetal, pieles de animales $\mathrm{o}$ aves, $\mathrm{y}$ un reducido ajuar $\mathrm{y}$ ofrendas funerarias ${ }^{15,17-19}$.

PLM-8PEC1 no fue momificado artificialmente pero presenta un tipo de entierro característico de la tradición Chinchorro y, de acuerdo con el fechado radiocarbónico, se sitúa temporalmente en el período Arcaico Tardío (5.000-3.700 A.P.) o Chinchorro Tardío, coincidente con un proceso 


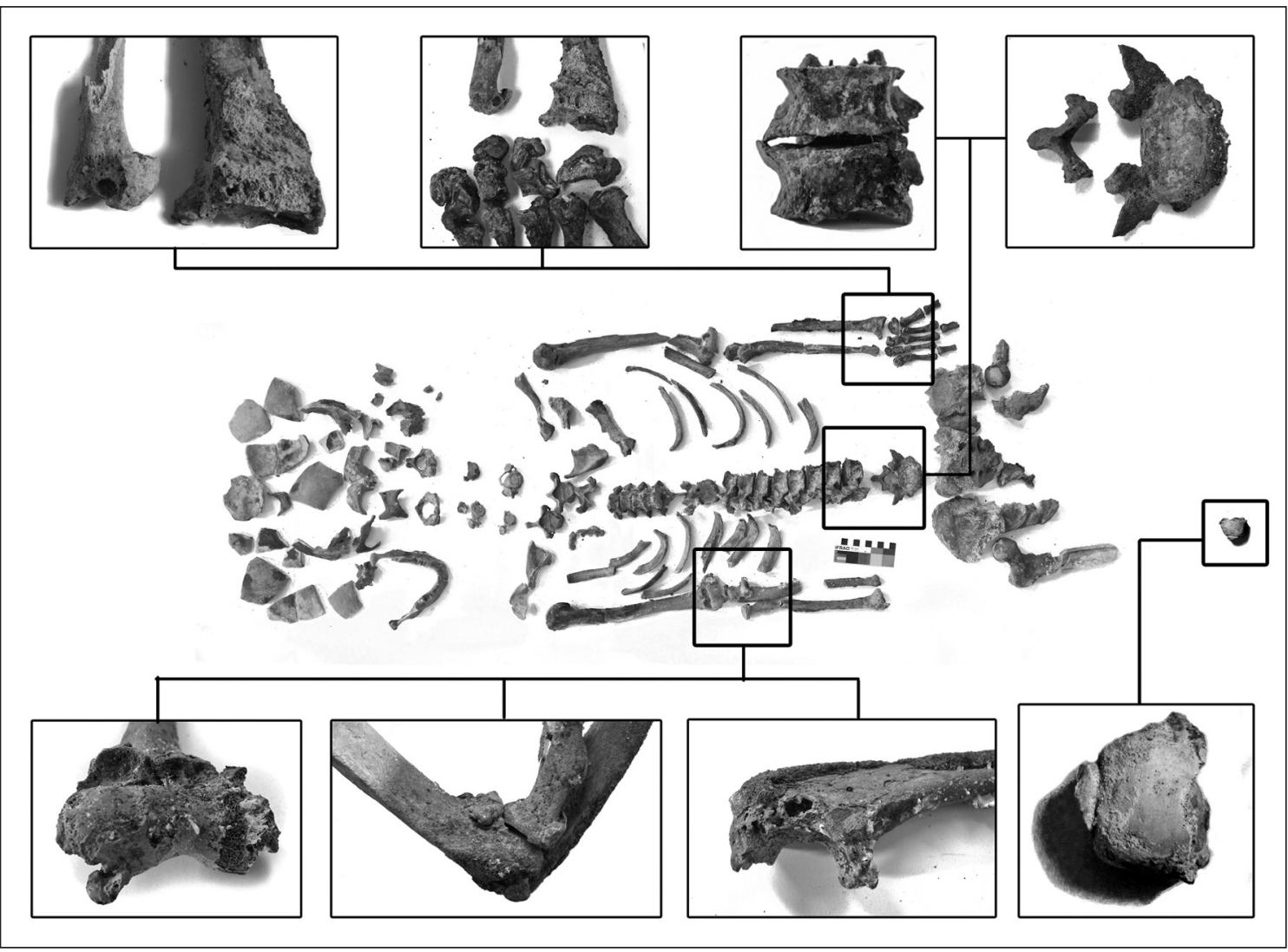

Figura 3.

gradual de transición desde un modo de vida esencialmente cazador- recolector a uno productor de alimentos; y coincidente también con el abandono paulatino de la práctica de la momificación artificial $^{20-25}$.

Un caso contemporáneo y similar a PLM8PEC1 es señalado en el sitio arqueológico Morro $1^{15}$. Se trata de M1T27C4, un individuo masculino de unos 35 años de edad, con lesiones erosivas simétricas bien definidas en la mayoría de sus articulaciones, incluyendo las manos. Ambos destacan como casos excepcionales, ya que en Chinchorro las artropatías son con frecuencia monoarticulares y vertebrales. En efecto, Standen $y$ colaboradore ${ }^{25}$ reportan en el sitio Morro 1 una ocurrencia de espondilosis y espondiloartrosis de $38 \%$ (14/37) y artropatías mono o biarticulares en las articulaciones periféricas en 18\% (12/65). Para los autores las causas se relacionan a actividades de subsistencia como caza, pesca, recolección, carga y procesamiento de alimentos. En este con- texto, Arriaza ${ }^{15}$ señala que en el caso de M1T27C4 las lesiones articulares le habrían provocado al individuo una discapacidad paulatina hasta la completa dependencia de su grupo social, lo que en sus palabras demuestra el "lado caritativo de la sociedad Chinchorro".

La presencia de exostosis auditiva externa en PLM-8PEC1 indica que este individuo fue económicamente activo durante gran parte de su vida, pues se postula que esta patología tiene un origen reactivo frente a agresiones externas como la exposición a aguas frías ${ }^{26,27}$. Se deduce que en vida el individuo practicó el nado y buceo para la pesca y recolección marina ${ }^{15,28}$. Estas actividades probablemente se vieron mermadas con el desarrollo de la enfermedad articular identificada, debido a la pérdida gradual de la movilidad, hipertrofia y crecimiento de osteofitos que, en el caso del codo derecho, terminaron por bloquear la articulación, impidiendo el movimiento de flexión y extensión del antebrazo derecho. 
Respecto a la dentadura, Kennedy ${ }^{29}$ plantea que el desgaste severo en incisivos y la pérdida de piezas en sociedades pescadoras se relacionan con actividades de manufactura de cabos y redes de pesca. Por lo que en este caso la condición dental se relacionaría al uso parafuncional de la dentadura, que supliría la pérdida progresiva de motricidad fina de las manos debido a la rigidez, dolor e inflamación articular.

En cuanto al diagnóstico diferencial de la patología articular, la edad del individuo, las características de las lesiones y su localización, indican un cuadro de carácter secundario ${ }^{5}$. Para la prehistoria de Arica se ha comprobado la presencia de patologías metabólicas como la osteoporosis y probables cuadros de anemia, infecciones como la tuberculosis, treponematosis, zoonosis y osteomielitis; traumas, tumores y deformaciones congénitas $^{8,15,25}$, a lo que se incluyen intoxicaciones crónicas por metales pesados como el arsénico ${ }^{30,31}$. Cada una de estas enfermedades podría tener como resultado la afectación de las articulaciones, sin embargo, la exostosis periarticular y principalmente la presencia de abscesos se relaciona con un proceso infeccioso.

La mayoría de los casos de artritis séptica son causados por estreptococos, estafilococos, Mycobacterium tuberculosis, Cándida albicans, Salmonella, Brucella, Neisseria y algunos virus. Estas infecciones se diseminan vía hematógena y acceden a la cavidad articular ocasionando inflamación, destrucción del cartílago articular y consecuentemente del tejido óseo ${ }^{32}$. De todas formas no puede descartarse que la infección en las articulaciones sea a su vez una complicación de una enfermedad prexistente como la artritis reumatoide o un cuadro sistémico.

En grupos de cazadores recolectores altamente móviles, la presencia de patologías severas debió tener importantes consecuencias en sus dinámicas sociales ${ }^{33}$. No obstante, los grupos Chinchorro manifestaron bajos niveles de movilidad, facilitado por la estabilidad y diversidad de recursos que ofrece el mar $^{34}$. En este contexto el caso PLM-8PEC1 contribuye a relativizar el entendimiento de la discapacidad -entendida como la pérdida de alguna función física que conlleva limitaciones permanentes- en sociedades cazadoras recolectoras, en las cuales las limitaciones físicas no necesariamente implicaron un castigo social. Lo que se sostiene desde el punto de vista cultural en la relatividad del concepto de acuerdo a las concepciones existentes en un determinado sistema social ${ }^{3,35}$.

El estado de salud del presente caso de estudio demuestra que hacia el final de su vida el individuo presentaba cuadros de otitis e hipoacusia por la exostosis auditiva externa ${ }^{28}$, era edentado (los dientes presentes sólo conservaban la raíz), sufría de dolores articulares crónicos y movilidad limitada, principalmente en la columna lumbar, muñecas, rodillas y codos, el derecho con incapacidad de flexión y extensión del antebrazo. Lo que en resumen le impedía realizar actividades de fuerza y destreza física y manual, y por tanto, contribuir a la subsistencia de su grupo social e inclusive de sí mismo ${ }^{4,5}$. Esto lleva a plantear que necesariamente PLM-8PEC1 dependió, al menos en la última etapa de su vida, de otros para sobrevivir.

Esta dependencia no representa en los conceptos de autocuidado un gesto caritativo de ayuda unidireccional a los necesitados, sino que corresponde a una respuesta adaptativa propia del ser humano que es simétrica y bidireccional, estableciéndose una relación de interdependencia que parte desde la aceptación del otro como igual ${ }^{36}$. Con ello se reafirman dinámicas y actitudes sociales particulares en esta sociedad Chinchorro relacionada con el autocuidado de los enfermos y discapacitados.

Como parte del rito mortuorio, PLM-8PEC1 fue cubierto de pigmento de color rojo en la cabeza y tronco, lo que permite reforzar la idea de pertenencia y cuidado de parte de su grupo social. El color, en efecto, es producto de conocimientos específicos manifestados desde la obtención de las materias primas hasta la preparación de la pintura y su aplicación ${ }^{37}$. Dado su uso restringido en los entierros arcaicos, el color se relacionaría con prácticas sociales específicas tendientes a diferenciar los individuos de acuerdo a determinados roles, relaciones intra e intergrupos. Así, el tratamiento del cuerpo tras la muerte del individuo refuerza su pertenencia a la sociedad Chinchorro, la que proponemos desarrolló y mantuvo dinámicas sociales específicas en relación al autocuidado ${ }^{38}$.

Agradecimientos: Al Dr. Robinson Silva e Indira Montt por sus aportes y correcciones al manuscrito, a Manuel Alarcón por la edición de las Figuras, al Proyecto FONDECYT 1100354, Beca de Doctorado Nacional 2010 CONICYT N 21100628 
y MECESUP UTA 0801 y al Convenio de Desempeño Universidad de tarapacá-MINEDUC. Finalmente a los editores y evaluadores por sus correcciones y aportes al artículo.

\section{Referencias}

1. Harris M. Antropología Cultural. Alianza Editorial 1998.

2. Wua X-J, Lynne Schepartza L, A, Liu W, Trinkausc E. Antemortem trauma and survival in the late Middle Pleistocene human cranium from Maba, South China. www.pnas.org/cgi/doi/10.1073/pnas.1117113108.

3. Arenas-Montreal L, Jasso-Arenas J, Campos-Navarro R. Autocuidado: elementos para sus bases conceptuales. Glob Health Promot 2011; 18: 42-8.

4. Quintero M, Monfort J, Mitrovic DR. Osteoartorsis. Editorial Médica Panamericana 2010.

5. Sociedad Española de Reumatología. Artrosis. Fisiopatología, diagnóstico y tratamiento. Editorial Médica Panamericana 2010.

6. Bridges P. Prehistoric arthritis in the Americas. Annu Rev Anthropol 1992; 21: 67-91.

7. Weiss E, Jurmain R. Osteoarthritis revisited: a contemporary review of a etiology. Int J Osteoarch 2007; 17: 437-50.

8. Aufderheide A, C Rodríguez-Martin. The Cambridge encyclopedia of human paleopathology. Cambridge UK: Cambridge University Press 1998.

9. Ortner D. Identification of pathological conditions in human skeletal remains. San Diego, EEUU: Academic Press 2003.

10. Jurmain Rd, Kilgore L. Skeletal evidence of osteoarthritis: A palaeopathological perspective. Ann Rheum Dis 1995; 54: 443-450.

11. Alvarez L. Un cementerio precerámico con momias de preparación complicada. Rehue 1969; 2: 181-90.

12. Buikstra, J. y D. Ubelaker. 1994 Standards for data collection from human skeletal remains. Arkansas Archaeological Survey, Research Series $\mathrm{N}^{\circ} 44$.

13. Núñez L. Paleoindio y arcaico en Chile: Diversidad, secuencia y proceso. México: Ediciones Cuicuilco 1983.

14. Núñez L. Registro regional de fechas radiocarbónicas del norte de Chile. Atacameños 1976; 4: 69-111.

15. Arriaza B. Cultura Chinchorro, las momias artificiales más antiguas del mundo. Editorial Universitaria 2003.

16. Muñoz I. Las sociedades costeras en el litoral de Arica durante el período arcaico tardío y sus vinculaciones con la costa peruana. Chungará 1982; 9: 124-51.

17. Standen V, C. Santoro y B. Arriaza. Síntesis y propuestas para el período arcaico en la costa del extremo norte de
Chile. Chungará 2004; 36: 201-12.

18. Rivera M. Hacia la complejidad social y política: el desarrollo Alto Ramírez del norte de Chile. Diálogo Andino 1994; 13: 9-38.

19. Standen V. Temprana complejidad funeraria en la cultura Chinchorro: (norte de Chile). Lat Am Antiq 1997; 8: 134-56.

20. Arriaza B. Tipología de las momias chinchorro y evolución de las prácticas de momificación. Chungará 1994; 26: 11-48.

21. Dauelsberg P. Excavaciones arqueológicas en Quiani (Provincia de Tarapacá, Departamento de Arica). Chungará 1974; 4: 61-3.

22. Niemeyer H, Schiappacasse V. Investigaciones arqueológicas en las terrazas de Conanoxa, valle de Camarones (Provincia de Tarapacá). Rev Universitaria 48, Anales de la Academia Chilena de Ciencias Naturales 1963; 26: 101-66.

23. Núñez L. Archaic adaptation on the south Central Andean coast. En: M. Blake, Editor, Pacific Latin America in Prehistory: The evolution of archaic and formative cultures. Washington, EEUU: University Press 1999; 199-212.

24. Núñez L, Santoro C. El tránsito arcaico-formativo en la circumpuna y valles occidentales del centro sur andino: hacia los cambios "Neolíticos". Chungará 2011; 43: (1) 487-530.

25. Standen V, Allison M, Arriaza B. Patologías óseas en la población Morro-1, asociada al Complejo Chinchorro: norte de Chile. Chungará 1984; 13: 175-85.

26. Granell J, Puig A, Benito E. Osteoma y exóstosis del conducto auditivo externo: un diagnóstico clínico. Acta Otorrinolaringol Esp 2003; 54: 229-32.

27. Altuna X, Vea JC, Camacho JJ, Algaba J. Tratamiento quirúrgico de las exóstosis de conducto auditivo externo. Acta Otorrinolaringol Esp 2006; 57: 257-61.

28. Standen V, Arriaza B, Santoro C. External Auditory Exostosis in Prehistoric Chilean Populations: A Test of the Cold Water Hypothesis. Am J Phys Anthropol 1997; 103: 119-29.

29. Kennedy K. Skeletal Markers of Life From the Skeleton. En: Iscan M, Kennedy K. Reconstruction of life from the skeleton. Alan R. Liss Inc. New York 1989: 129-60.

30. Arriaza B. Arseniasis as an environmental hypothetical explanation for the origin of the oldest artificial mummification practice in the world. Chungará 2005; 37: 255-60.

31. Silva-Pinto V, Arriaza A, Standen V. Evaluación de la frecuencia de espina bífida oculta y su posible relación con el arsénico ambiental en una muestra prehispánica de la Quebrada de Camarones, norte de Chile. Rev Med Chile 2010; 138: 461-9. 
Un caso de osteoartropatía en la prehistoria de Arica - V. Silva-Pinto et al

32. Ross JO. Septic arthritis. Infect Dis Clin N Am 2005: 19: 799-817.

33. Luna LH, Aranda CM, Bosio LA, Berón MA. A Case of Multiple Metastasis in Late Holocene Hunter-Gatherers from the Argentine Pampean Region. Int J Osteoarchaeol 2008; 18: 492-506.

34. Marquet P, Santoro C, Latorre C, Standen V, Abadesa S, Rivadeneira M, et al. Emergence of social complexity among coastal hunter-gatherers in the Atacama desert of northern Chile. PNAS 2012: 1-7. doi:10.1073/ pnas.1116724109.

35. Juárez F, Holguín E, Salamanca A. Aceptación o rechazo: perspectiva histórica sobre la discapacidad, la rehabilitación y la psicología de la rehabilitación. Psicología y Salud 2006; 16: 187-97.

36. Urra E, Jana A, García M. Algunos aspectos esenciales del pensamiento de Jean Watson y su teoría de cuidados transpersonales. Cienc. Enferm 2011; 17 (3): 11-22.

37. Sepúlveda M. Pinturas rupestres y tecnología del color en el extremo sur de Chile. Magallania 2011; 39 (1): 195212.

38. Spikins P, Rutherford H, Needham A. From Hominity to Humanity: compassion from the earliest archaics to modern humans. Time and Mind 2010; 3 (3): 303-25. 